\title{
Phân tích phẩm chất, dung lượng hệ thống diều chế không gian song công trên cùng băng tần
}

\author{
Nguyễn Lê Vân, Nguyễn Bá Cao, Trần Xuân Nam \\ Học viện Kỹ thuật Quân sự, Hà Nội \\ Correspondence: Nguyễn Lê Vân, vannl@mta.edu.vn \\ Ngày nhận bài: 17/08/2019, ngày sửa chữa: 07/09/2019, ngày duyệt đăng: 08/09/2019 \\ Xem sớm trực tuyến: 13/09/2019, định danh DOI: 10.32913/mic-ict-research-vn.v2019.n1.883 \\ Biên tập lĩnh vực điều phối phản biện và quyết định nhận đăng: TS. Nguyễn Việt Dũng
}

Tóm tắt: Bài báo này phân tích phẩm chất và dung lượng hệ thống đa đầu vào - đa đầu ra sử dụng kỹ thuật điều chế không gian và truyền dẫn song công trên cùng băng tần. Chúng tôi đã xác định biểu thức chính xác của xác suất dừng, xác suất lỗi ký hiệu và dung lượng trung bình của hệ thống khảo sát. Trên cơ sở đó, phẩm chất hệ thống đã được phân tích, đánh giá toàn diện dưới sự ảnh hưởng của nhiễu dư do hoạt động của truyền dẫn song công gây nên. Đồng thời kết quả được so sánh với hệ thống đa đầu vào - đa đầu ra kết hợp điều chế không gian truyền thống (hệ thống bán song công). Cuối cùng, sự đúng đắn của kết quả phân tích được kiểm chứng bằng mô phỏng Monte-Carlo.

Từ khóa: Đa đầu vào - đa đầu ra, điều chế không gian, truyền thông song công trên cùng băng tần, triệt nhiếu tư giao thoa, xác suất dùng, xác suất lỗi ký hiệu, dung lượng ergodic.

Title: Performance and Capacity Analysis of Spatial Modulation Full Duplex Systems

Abstract: In this paper, we analyze the performance and ergodic capacity of multiple input-multiple output (MIMO) system using spatial modulation (SM) and the full-duplex (FD) technique. Based on the numerical analysis, we derive exact expressions of the outage probability, the symbol error probability and the ergodic capacity of the considered system. From the theoretical analysis, the system performance is analyzed fully under the impact of residual self-interference due to the FD mode. On the other hand, the numerical results are compared with the traditional MIMO-SM system (in half-duplex). Finally, numerical results are verified by Monte-Carlo simulations.

Keywords: Multiple input-multiple output, spatial modulation, full-duplex, outage probability, symbol error probability, ergodic capacity.

\section{I. ĐặT VẤN ĐỀ}

Điều chế không gian (SM: Spatial Modulation) là sơ đồ truyền dẫn hứa hẹn cho hệ thống truy nhập đa đầu vào đa đầu ra (MIMO: Multiple-Input Multiple-Output) bởi kỹ thuật này cho phép giảm độ phức tạp và chi phí thiết kế cho hệ thống MIMO trong khi vẫn giữ nguyên phẩm chất và tốc độ truyền dẫn [1-3]. Do đó, trong những năm gần đây hệ thống MIMO-SM đã trở thành chủ đề nhận được nhiều sự quan tâm của các nhà nghiên cứu và thiết kế mạng vô tuyến, chẳng hạn như trong các nghiên cứu $[1,2,4-7]$.

Tác giả trong công trình $[2,4]$ đã đề xuất các bộ tách tín hiệu có độ phức tạp thấp cho máy thu SM, giảm được độ phức tạp đáng kể so với máy thu MIMO truyền thống. Trong công trình [5-7] các tác giả đã xem xét phẩm chất và hiệu quả phổ tần của hệ thống MIMO-SM trong các điều kiện khác nhau. Tác giả trong [5] đã thiết kế và đánh giá phẩm chất hệ thống MIMO-SM sử dụng đơn sóng mang thông qua cấu hình ăng-ten. Kết quả cho thấy mô hình đề xuất đã tăng hiệu quả phổ và giảm độ phức tạp tính toán. Trong công trình [6] tác giả đã đề xuất quá trình tiền xử lý để truyền dẫn qua kênh tương quan thông qua thông tin trạng thái kênh (CSI: Channel State Information) ở máy phát, nhờ đó phẩm chất hệ thống MIMO-SM đã được cải thiện đáng kể. Để đánh giá hiệu quả sử dụng phổ tần, công trình [7] đã đưa ra biểu thức xấp xỉ dung lượng và xác suất lỗi hệ thống MIMO-SM, từ đó đánh giá toàn diện hệ thống khảo sát.

Bên cạnh đó, trong giai đoạn bùng nổ về truyền thông và công nghệ thông tin hiện nay, nhu cầu trao đổi dữ liệu tốc độ cao ngày càng tăng, đòi hỏi cần phải nâng cao hiệu quả sử dụng phổ tần. Đứng trước bối cảnh đó, nhiều kỹ thuật mới đã được đề xuất, trong đó kỹ thuật truyền thông song công trên cùng băng tần (FD: Full-Duplex hay IBFD: In-Band Full-Duplex) đang được chú trọng bởi khả năng tăng gấp đôi hiệu quả sử dụng phổ tần do cùng thu và phát trên cùng một tần số và tại cùng một thời điểm [8-13]. Đặc biệt kỹ thuật FD càng trở nên khả thi hơn khi nhiều nghiên 
cứu và thử nghiệm cho thấy thiết bị FD có thể khử nhiễu tự giao thoa (SIC: Self-Interference Cancellation) lên đến $110 \mathrm{~dB}$ và đưa nhiễu này về mức nhiễu nền.

Gần đây, nhiều nghiên cứu đã kết hợp hệ thống MIMO$\mathrm{SM}$ với kỹ thuật $\mathrm{FD}$ tạo thành hệ thống $\mathrm{SM}-\mathrm{FD}$ nhằm khai thác những ưu điểm nổi trội của những kỹ thuật này [1417]. Trong [14] nhóm tác giả đã đưa ra biểu thức xác suất dừng và dung lượng Ergodic của hệ thống SM-FD điểmđiểm (point-to-point) để khảo sát hệ thống. Biểu thức này có thể áp dụng được cho trường hợp $2 \times 2 \mathrm{MIMO}$. Khi thiết bị đầu cuối có số lượng ăng-ten nhiều hơn, biểu thức tìm được ở [14] không còn áp dụng được. Để tăng cường độ tin cậy thông tin, mở rộng vùng phủ sóng, mạng chuyển tiếp sử dụng kỹ thuật $\mathrm{SM}$ và $\mathrm{FD}$ đã được nghiên cứu trong [15-17]. Các tác giả đã tìm ra biểu thức biên trên cho tỉ lệ lỗi bít (BER: Bit Error Rate) và so sánh với hệ thống bán song công (HD: Half-Duplex) khi nút chuyển tiếp sử dụng giao thức khuếch đại và chuyển tiếp ( $\mathrm{AF}$ : Amplifyand-Forward) [15]. Đồng thời, biểu thức xấp xỉ về $B E R$ và dung lượng Ergodic đã được xác định cho mạng chuyển tiếp SM-FD trong $[16,17]$. Thông qua kêt quả mô phỏng và biểu thức tiệm cận tìm được, các tác giả đã chứng minh được rằng hệ thống $\mathrm{SM}-\mathrm{FD}$ có thể đạt phẩm chất gần tương đương so với hệ thống SM truyền thống (SM-HD) khi nhiễu dư (RSI: Residual Self-Interference) rất nhỏ, trong khi dung lượng đạt cao hơn hệ thống SM-HD với một số mức ngưỡng nhất định.

Mặc dù phẩm chất của hệ thống SM-FD đã được quan tâm đánh giá thông qua nhiều công trình tiên phong như đã phân tích ở trên. Tuy nhiên, các kết quả công bố trước đây mới dừng lại ở việc tìm được các biểu thức về phẩm chất hệ thống ở dạng biểu thức tiệm cận hoặc thực hiện đánh giá phẩm chất bằng kết quả mô phỏng thông qua kết quả trung bình thống kê. Điều này dẫn đến hạn chế về sự hiểu biết chính xác hoạt động của hệ thống trong các điều kiện đầy đủ. Trong bài báo này chúng tôi đặt mục tiêu phát triển một khung toán học chính xác phục vụ cho việc phân tích, đánh giá đầy đủ hoạt động của hệ thống SM-FD.

Những đóng góp của bài báo được tóm tắt như sau:

- Bài báo tìm ra biểu thức chính xác về xác suất dừng (OP: Outage Probability), xác suất lỗi ký hiệu (SEP: Symbol Error Probability) và dung lượng trung bình (ergodic capacity) của hệ thống SM-FD trên kênh phađinh Rayleigh.

- Trên cơ sở các biểu thức giải tích chính xác, chúng tôi đánh giá phẩm chất hệ thống SM-FD khi so sánh với hệ thống SM-HD. Kết quả cho thấy, khi RSI đủ nhỏ hệ thống SM-FD cho phẩm chất tương đương hệ thống SM-HD trong khi dung lượng đạt được gần gấp đôi. Ngược lại, khi RSI lớn hơn, phẩm chất hệ thống SM-FD giảm so với hệ thống SM-HD trong khi dung lượng vẫn cao hơn tùy thuộc vào giá trị cụ thể của RSI và tỉ số tín hiệu trên tạp âm. Kết quả phân tích là cơ sở để tiếp tục nghiên cứu, thử nghiệm và đánh giá phẩm chất hệ thống SM-FD trước khi triển khai, áp dụng hệ thống trong thực tế.

Phần còn lại của bài báo được tổ chức như sau: trong Mục II chúng tôi trình bày mô hình hệ thống và tín hiệu. Mục III thể hiện những kết quả biến đổi toán học để đưa ra biểu thức về phẩm chất và dung lượng hệ thống. Mục IV trình bày kết quả mô phỏng và thảo luận, và cuối cùng, Mục $\mathrm{V}$ rút ra các kết luận của nghiên cứu.

\section{MÔ HÌNH Hệ THỐNG}

Mô hình hệ thống SM-FD được thể hiện trên hình 1 . Ở đây, chúng tôi sử dụng ăng-ten thu phát riêng cho mỗi thiết bị đầu cuối để biểu thị rõ nhiễu tự giao thoa (SI: SelfInteference), đồng thời tạo khả năng SIC tốt hơn cho thiết bị FD $[18,19]$. Tuy nhiên trong thực tế, thiết bị FD có thể sử dụng ăng-ten chung cho cả thu và phát. Dữ liệu được truyền đồng thời từ đầu cuối $\mathrm{A}$ sang đầu cuối $\mathrm{B}$, trong đó $\mathrm{A}$ và $\mathrm{B}$ có số ăng-ten phát và thu tương ứng là $N_{t}^{\mathrm{A}}, N_{r}^{\mathrm{A}}$ và $N_{t}^{\mathrm{B}}, N_{r}^{\mathrm{B}}$. Trên hình 1 , mũi tên màu xanh thể hiện tín hiệu phát, thu cần truyền và nhận, mũi tên màu đỏ thể hiện nhiễu tự giao thoa SI.

Tại thời điểm $t$ tín hiệu thu được tại đầu cuối $\mathrm{B}$ được xác định như sau:

$$
\mathbf{y}_{\mathrm{B}}(t)=\sqrt{P_{\mathrm{A}}} \mathbf{h}_{i}^{\mathrm{B}} x_{i}(t)+\sqrt{P_{\mathrm{B}}} \mathbf{h}_{j}^{\mathrm{B}} x_{j}(t)+\mathbf{z}_{\mathrm{B}}(t),
$$

trong đó $\mathrm{y}_{\mathrm{B}}$ là véc tơ tín hiệu thu, $P_{\mathrm{A}}$ và $P_{\mathrm{B}}$ lần lượt là công suất phát tại đầu cuối $\mathrm{A}$ và $\mathrm{B}, x_{i}$ và $x_{j}$ lần lượt là tín hiệu phát tại ăng-ten được kích hoạt thứ $i$ của đầu cuối $\mathrm{A}$ và thứ $j$ của đầu cuối $\mathrm{B}, \mathbf{h}_{i}^{\mathrm{B}}$ là véc tơ kênh truyền từ ăng-ten được kích hoạt thứ $i$ ở đầu cuối $\mathrm{A}$ tới $N_{r}^{\mathrm{B}}$ ăng-ten thu tại đầu cuối $\mathrm{B}, \mathbf{h}_{j}^{\mathrm{B}}$ là véc tơ kênh nhiễu tự giao thoa từ ăng-ten phát được kích hoạt thứ $j$ của đầu cuối B đến $N_{r}^{\mathrm{B}}$ ăng-ten thu của chính nó, $\mathbf{z}_{\mathrm{B}}$ là véc tơ tạp âm trắng cộng tính (AWGN: Additive White Gaussian Noise) với các phần tử có trung bình bằng không và phương sai $\sigma^{2}$.

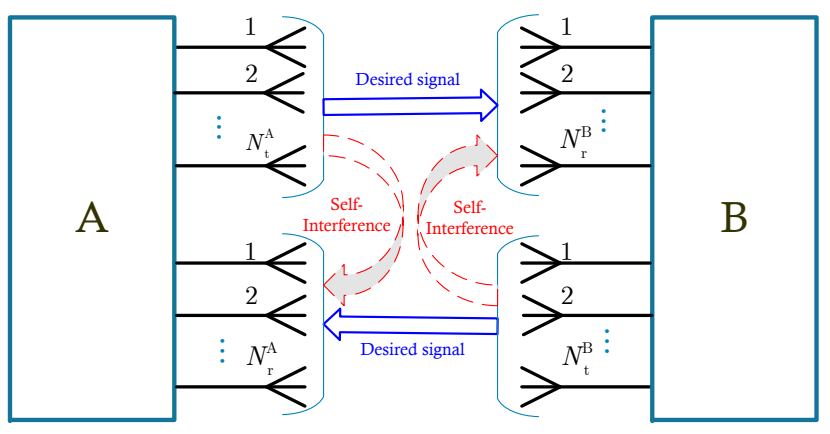

Hình 1. Mô hình hệ thống SM-FD. 
Để hệ thống FD có thể hoạt động theo yêu cầu thì cần phải triệt nhiễu SI một cách hiệu quả. Giải pháp đã được đề xuất sử dụng trong các công trình tiên phong về FD là sử dụng đồng thời kỹ thuật SIC trên cả 3 miền: miền không gian (hay miền ăng-ten), miền tương tự và miền số $[9,18,20]$. Giải pháp triệt nhiễu trên miền không gian liên quan đến các phương pháp ngăn cách tín hiệu phát vào tuyến thu như sử dụng 2 ăng-ten thu phát riêng biệt đặt cách xa nhau kết hợp ăng-ten định hướng, bọc kim phần phát và phần thu, sử dụng thay đổi phân cực, v.v. Nhờ vậy, có thể giảm đáng kể ảnh hưởng SI từ kênh trực tiếp. Bài báo [20] cho thấy có thể triệt nhiễu tới $50 \mathrm{~dB}$ trên miền không gian. Với các giải pháp triệt nhiễu trên miền không gian hiệu quả hơn nữa thì ảnh hưởng của kênh SI sẽ bị giới hạn chủ yếu bởi kênh phản xạ. Trong trường hợp này cường độ đường trực tiếp không còn vượt trội so với các tia phản xạ. Do các tia phản xạ và đường trực tiếp là độc lập thống kê với nhau nên theo định lý giới hạn trung tâm, độ lợi kênh SI tổng hợp sẽ trở thành một biến Gauss phức và kênh SI trở thành kênh pha-đinh Rayleigh. Kết quả khảo sát trong [4] cho thấy đặc tính của kênh SI trong phần lớn các trường hợp khảo sát có phân bố Rayleigh.

Sử dụng các giải pháp triệt nhiễu trên miền tương tự và miền số có thể cho phép đạt được thêm $45 \mathrm{~dB}$ trên miền tương tự và $50 \mathrm{~dB}$ trên miền số [20]. Cũng theo [20] thì nếu đạt được mức suy hao trên $110 \mathrm{~dB}$ thì đã cho phép chuyển nhiễu SI về mức nền tạp âm. Điều này đồng nghĩa với ảnh hưởng của RSI có thể coi như một biến tạp âm. Mặc dù kênh SI sau triệt nhiễu trên miền không gian có đặc tính Rayleigh nhưng do thao tác triệt nhiễu trên miền tương tự và miền số sử dụng phương pháp tái tạo lại tín hiệu thu được trên kênh truyền SI (thông qua ước lượng kênh truyền SI) để triệt nhiễu nên phần nhiễu dư RSI còn lại chỉ là phần sai số ước lượng. Sai số ước lượng này có thể được mô hình hóa bởi một biến ngẫu nhiên phân bố chuẩn như đã chỉ ra trong các nghiên cứu trước [20-22]. Như vậy, thành phần nhiễu $\sqrt{P_{\mathrm{B}}} \mathbf{h}_{j}^{\mathrm{B}} x_{j}(t)$ trong công thức (1) có thể viết lại thành véc tơ $\mathbf{r}_{\mathrm{SI}}$ với các phần tử theo phân bố chuẩn với trung bình bằng không và phương sai $\sigma_{\mathrm{RSI}}^{2}$, trong đó $\sigma_{\mathrm{RSI}}^{2}=\tilde{\Omega} P_{\mathrm{B}}$. Ở đây $\tilde{\Omega}$ là tỉ số giữa công suất đầu vào và đầu ra kênh SI thể hiện khả năng triệt nhiễu SI của thiết bị FD. Do đó, biểu thức (1) được viết lại thành

$$
\mathbf{y}_{\mathrm{B}}(t)=\sqrt{P_{\mathrm{A}}} \mathbf{h}_{i}^{\mathrm{B}} x_{i}(t)+\mathbf{r}_{\mathrm{SI}}(t)+\mathbf{z}_{\mathrm{B}}(t) .
$$

Tỉ số tín hiệu trên nhiễu cộng tạp âm (SINR: Signal to Interference plus Noise Ratio) tức thời của tín hiệu thu tại đầu cuối $\mathrm{B}$ được xác định như sau:

$$
\gamma_{\mathrm{B}}=\frac{\left\|\mathbf{h}_{i}^{\mathrm{B}}\right\|^{2} P_{\mathrm{A}}}{\sigma_{\mathrm{RSI}}^{2}+\sigma^{2}}=\left\|\mathbf{h}_{i}^{\mathrm{B}}\right\|^{2} \bar{\gamma}_{\mathrm{B}},
$$

trong đó $\bar{\gamma}_{\mathrm{B}}=P_{\mathrm{A}} /\left(\sigma_{\mathrm{RSI}}^{2}+\sigma^{2}\right)$ là SINR trung bình của tín hiệu thu được tại đầu cuối $\mathrm{B}$.
Biểu thức tín hiệu thu tại đầu cuối $\mathrm{A}$ được xác định bằng phương pháp tương tự.

Chú ý rằng, khi kênh truyền là kênh pha-đinh Rayleigh, hàm phân bố tích lũy (CDF: Cumulative Distribution Function) và hàm mật độ xác suất (PDF: Probability Density Function) của độ lợi kênh truyền tức thời được xác định như sau:

$$
\begin{gathered}
F_{|h|^{2}}(x)=1-\exp \left(-\frac{x}{\Omega}\right), x \geq 0 \\
f_{|h|^{2}}(x)=\frac{1}{\Omega} \exp \left(-\frac{x}{\Omega}\right), x \leq 0
\end{gathered}
$$

trong đó $\Omega=\mathbb{E}\left\{|h|^{2}\right\}$ là độ lợi trung bình của kênh truyền, toán tử $\mathbb{E}$ biểu diễn phép lấy kỳ vọng.

\section{PHÂN TÍCH PHẨM CHẤT HÊ THỐNG}

\section{Xác suất dừng hệ thống SM-FD}

Xác suất dừng hệ thống được xác định bằng xác suất mà giá trị SINR nhỏ hơn một mức ngưỡng đã xác định trước [23]. Giả sử tốc độ truyền dẫn của hệ thống là $\mathcal{R}$, xác suất dừng của hệ thống SM-FD được xác định bằng biểu thức sau [24-26]:

$$
\begin{aligned}
\mathcal{P}_{\text {out }} & =\operatorname{Pr}\left\{\log _{2}\left(N_{t}\right)+\log _{2}(1+\gamma)<\mathcal{R}\right\} \\
& =\operatorname{Pr}\left\{\gamma<2^{\mathcal{R}-\log _{2}\left(N_{t}\right)}-1\right\},
\end{aligned}
$$

trong đó, Pr biểu diễn phép lấy xác suất (Probability), $\gamma$ là SINR tức thời tại đầu cuối xem xét $\left(\gamma_{\mathrm{A}}\right.$ tại phía $\mathrm{A}$ và $\gamma_{\mathrm{B}}$ tại phía $\mathrm{B}), N_{t}$ là số ăng-ten phát $\left(N_{t}^{\mathrm{A}}\right.$ ở phía $\mathrm{A}$ và $N_{t}^{\mathrm{B}}$ ở phía $\mathrm{B})$. Chú ý rằng, thành phần $\log _{2}\left(N_{t}\right)$ biểu thị kỹ thuật SM được áp dụng. Trong bài báo này, để tập trung đánh giá ảnh hưởng của RSI đến phẩm chất của hệ thống và để đơn giản cho việc phân tích, tương tự như trong [24-26] chúng tôi giả thiết rằng máy thu ước lượng hoàn hảo chỉ số ăng-ten phát, từ đó giải mã thành công tổ hợp bít dùng để kích hoạt ăng-ten phát . Trên cơ sở đó, ta xác định được xác suất dừng cho hệ thống SM-FD như định lý 1 dưới đây.

Định lý 1: Xác suất dừng tại đầu cuối $\mathrm{A}$ (ký hiệu là $\mathcal{P}_{\mathrm{out}}^{\mathrm{A}}$ ) và đầu cuối $\mathrm{B}$ (ký hiệu là $\mathcal{P}_{\text {out }}^{\mathrm{B}}$ ) của hệ thống $\mathrm{SM}-\mathrm{FD}$ lần lượt được xác định như sau:

$$
\begin{aligned}
& \mathcal{P}_{\text {out }}^{\mathrm{A}}=1-e^{-\frac{\gamma_{\text {th }}}{\bar{\gamma}_{\mathrm{A}}}} \sum_{i=0}^{N_{r}^{\mathrm{A}}-1} \frac{\left(\frac{\gamma_{\text {th }}}{\bar{\gamma}_{\mathrm{A}}}\right)^{i}}{i !}, \\
& \mathcal{P}_{\text {out }}^{\mathrm{B}}=1-e^{-\frac{\gamma_{\text {th }}}{\bar{\gamma}_{\mathrm{B}}}} \sum_{i=0}^{N_{r}^{\mathrm{B}}-1} \frac{\left(\frac{\gamma_{\text {th }}}{\bar{\gamma}_{\mathrm{B}}}\right)^{i}}{i !},
\end{aligned}
$$

trong đó $\gamma_{\mathrm{th}}=2^{\mathcal{R}-\log _{2}\left(N_{t}\right)}-1$ là mức ngưỡng xem xét, $\bar{\gamma}_{\mathrm{A}}$ và $\bar{\gamma}_{B}$ là SINR trung bình tại đầu cuối $A$ và $B$ tương ứng. 
Chứng minh: Đặt $\gamma_{\mathrm{th}}=2^{\mathcal{R}-\log _{2}\left(N_{t}\right)}-1$ biểu thị mức ngưỡng xem xét (threshold), biểu thức (6) trở thành:

$$
\mathcal{P}_{\text {out }}=\operatorname{Pr}\left\{\gamma<\gamma_{\text {th }}\right\} \text {. }
$$

Do đó, xác suất dừng tại $\mathrm{B}$ được xác định như sau:

$$
\mathcal{P}_{\text {out }}^{\mathrm{B}}=\operatorname{Pr}\left\{\frac{\left\|\mathbf{h}_{i}^{\mathrm{B}}\right\|^{2} P_{\mathrm{A}}}{\sigma_{\mathrm{RSI}}^{2}+\sigma^{2}}<\gamma_{\mathrm{th}}\right\}=\operatorname{Pr}\left\{\left\|\mathbf{h}_{i}^{\mathrm{B}}\right\|^{2}<\frac{\gamma_{\mathrm{th}}}{\bar{\gamma}_{\mathrm{B}}}\right\} .
$$

Từ (10), sau một số phép biến đổi, kết hợp với áp dụng biểu thức (4) và (5) cho trường hợp các kênh truyền có cùng độ lợi, ta xác định được xác suất dừng tại đầu cuối $\mathrm{A}$ và đầu cuối $\mathrm{B}$ lần lượt như biểu diễn trong định lý 1 . Chú ý rằng, biểu thức (7) và (8) có thể biểu diễn đơn giản hơn theo hàm Gamma, tuy nhiên biểu diễn theo dạng tổng sẽ thuận lợi hơn trong tính toán các tham số hệ thống tiếp theo.

\section{Xác suất lỗi ký hiệu}

Đối với hệ thống vô tuyến, xác suất lỗi ký hiệu (SEP: Symbol Error Probability) được xác định thông qua biểu thức sau [23]:

$$
\mathrm{SEP}=a\{Q(\sqrt{b \gamma})\}=\frac{a}{\sqrt{2 \pi}} \int_{0}^{\infty} F\left(\frac{t^{2}}{b}\right) e^{-\frac{t^{2}}{2}} d t
$$

trong đó toán tử $\mathbb{E}$ biểu diễn phép lấy kỳ vọng; $Q$ là hàm Gaussian; $\gamma$ là SINR tức thời của hệ thống khảo sát; $a, b$ là hằng số phụ thuộc vào dạng điều chế tín hiệu và được xác định theo bảng I [23].

Trên cơ sở đó, ta xác định được xác suất lỗi ký hiệu tại máy thu hệ thống SM-FD như ở định lý 2 .

Định lý 2: Xác suất lỗi ký hiệu tại đầu cuối $\mathrm{A}\left(\mathrm{SEP}_{\mathrm{A}}\right)$ và $\mathrm{B}\left(\mathrm{SEP}_{\mathrm{B}}\right)$ của hệ thống $\mathrm{SM}-\mathrm{FD}$ được xác định lần lượt thông qua các biểu thức sau:

$$
\begin{aligned}
& \mathrm{SEP}_{\mathrm{A}}=\frac{a}{2}-\frac{a \sqrt{b}}{2 \sqrt{2 \pi}} \sum_{i=0}^{N_{r}^{\mathrm{A}}-1} \frac{2^{i} \sqrt{2 \bar{\gamma}_{\mathrm{A}}} \Gamma\left(i+\frac{1}{2}\right)}{i !\left(2+\bar{\gamma}_{\mathrm{A}} b\right)^{\left(i+\frac{1}{2}\right)}}, \\
& \mathrm{SEP}_{\mathrm{B}}=\frac{a}{2}-\frac{a \sqrt{b}}{2 \sqrt{2 \pi}} \sum_{i=0}^{N_{r}^{\mathrm{B}}-1} \frac{2^{i} \sqrt{2 \bar{\gamma}_{\mathrm{B}}} \Gamma\left(i+\frac{1}{2}\right)}{i !\left(2+\bar{\gamma}_{\mathrm{B}} b\right)^{\left(i+\frac{1}{2}\right)}} .
\end{aligned}
$$

\section{Bảng I}

GIÁ TRI CỦA $a$ VÀ $b$ CHO CÁC DẠNG ĐIỀU CHÊ KHÁC NHAU

\begin{tabular}{|l|l|l|l|}
\hline Kiểu điều chế & SEP & $a$ & $b$ \\
\hline BFSK & $Q(\sqrt{\gamma})$ & 1 & 1 \\
\hline BPSK & $Q(\sqrt{2 \gamma})$ & 1 & 2 \\
\hline QPSK,4-QAM & $\approx 2 Q(\sqrt{\gamma})$ & 2 & 1 \\
\hline M-PAM & $\approx \frac{2(M-1)}{M} Q\left(\sqrt{\frac{6}{M^{2}-1}} \gamma\right)$ & $\frac{2(M-1)}{M}$ & $\frac{6}{M^{2}-1}$ \\
\hline M-PSK & $\approx 2 Q\left(\sqrt{2 \sin ^{2}\left(\frac{\pi}{M}\right) \gamma}\right)$ & 2 & $2 \sin ^{2}\left(\frac{\pi}{M}\right)$ \\
\hline M-QAM chữ nhật & $\approx \frac{4(\sqrt{M}-1)}{M} Q\left(\sqrt{\frac{3}{M-1}} \gamma\right)$ & $\frac{4(\sqrt{M}-1)}{M}$ & $\frac{3}{M-1}$ \\
\hline M-QAM không chữ nhật & $\approx 4 Q\left(\sqrt{\frac{3}{M-1}} \gamma\right)$ & 4 & $\frac{3}{M-1}$ \\
\hline
\end{tabular}

trong đó $\Gamma(\cdot)$ là hàm Gamma.

Chứng minh: Đặt $x=t^{2} / b$, sau một số phép biến đổi toán học, biểu thức (11) trở thành

$$
\mathrm{SEP}=\frac{a \sqrt{b}}{2 \sqrt{2 \pi}} \int_{0}^{\infty} \frac{e^{-b x / 2}}{\sqrt{x}} F(x) d x,
$$

với $F(x)$ là $\mathrm{CDF}$ theo SINR của hệ thống khảo sát. Trên cơ sở định nghĩa hàm $\mathrm{CDF}$, ta có thể thay thế $F(x)$ bằng $\mathcal{P}_{\text {out }}$ của hệ thống khi thay thế $\gamma_{\text {th }}$ trong $\mathcal{P}_{\text {out }}$ bởi $x$. Từ đó, ta tính toán xác suất lỗi tại đầu cuối $\mathrm{A}\left(\mathrm{SEP}_{\mathrm{A}}\right)$ thông qua biểu thức

$$
\mathrm{SEP}_{\mathrm{A}}=\frac{a \sqrt{b}}{2 \sqrt{2 \pi}}(\mathrm{I}-\mathrm{J})
$$

trong đó

$$
\begin{aligned}
& \mathrm{I}=\int_{0}^{\infty} \frac{e^{-b x / 2}}{\sqrt{x}} d x, \\
& \mathrm{~J}=\int_{0}^{\infty} \frac{e^{-b x / 2}}{\sqrt{x}} e^{-\frac{x}{\bar{\gamma}_{\mathrm{A}}}} \sum_{i=0}^{N_{r}^{\mathrm{A}}-1} \frac{\left(\frac{x}{\bar{\gamma}_{\mathrm{A}}}\right)^{i}}{i !} d x .
\end{aligned}
$$

Với thành phần I trong (15), áp dụng công thức (3.361.2) trong [27] ta thu được

$$
\mathrm{I}=\int_{0}^{\infty} \frac{e^{-b x / 2}}{\sqrt{x}} d x=\sqrt{\frac{2 \pi}{b}} .
$$

Với thành phần $\mathrm{J}$ trong biểu thức (15), áp dụng công thức (3.381.4) trong [27], ta có

$$
\begin{aligned}
\mathrm{J} & =\int_{0}^{\infty} \sum_{i=0}^{N_{r}^{\mathrm{A}}-1} \frac{1}{i ! \bar{\gamma}_{\mathrm{A}}^{i}} e^{-x\left(\frac{b}{2}+\frac{1}{\bar{\gamma}_{\mathrm{A}}}\right)} x^{i-\frac{1}{2}} d x \\
& =\sum_{i=0}^{N_{r}^{\mathrm{A}}-1} \frac{2^{i} \sqrt{2 \bar{\gamma}_{\mathrm{A}}} \Gamma\left(i+\frac{1}{2}\right)}{i !\left(2+\bar{\gamma}_{\mathrm{A}} b\right)^{\left(i+\frac{1}{2}\right)}} .
\end{aligned}
$$

Kết hợp (16) và (17) ta thu được biểu thức tường minh rút gọn cho xác suất lỗi tại $\mathrm{A}$ như (12). Bằng phương pháp tương tự, ta xác định được xác suất lỗi tại $\mathrm{B}$ như (13).

\section{Dung lượng Ergodic}

Với hệ thống song công hai chiều, dung lượng Ergodic được xác định thông qua biểu thức

$$
\begin{aligned}
C & =\mathbb{E}\left\{\log _{2}\left(1+\gamma_{\mathrm{A}}\right)+\log _{2}\left(1+\gamma_{\mathrm{B}}\right)\right\} \\
& =\mathbb{E}\left\{\log _{2}\left(1+\gamma_{\mathrm{A}}\right)\right\}+\mathbb{E}\left\{\log _{2}\left(1+\gamma_{\mathrm{B}}\right)\right\} .
\end{aligned}
$$

Từ đó ta được

$$
C=\frac{1}{\ln 2} \int_{0}^{\infty} \frac{1-F_{\mathrm{A}}(x)}{1+x} d x+\frac{1}{\ln 2} \int_{0}^{\infty} \frac{1-F_{\mathrm{B}}(x)}{1+x} d x,
$$


trong đó $F_{\mathrm{A}}(x)$ và $F_{\mathrm{B}}(x)$ lần lượt là $\mathrm{CDF}$ tại đầu cuối $\mathrm{A}$ và $\mathrm{B}$. Trên cơ sở đó, dung lượng hệ thống được xác định qua định lý 3 dưới đây.

Định lý 3: Dung lượng Ergodic hệ thống SM-FD được xác định như sau:

$$
\begin{aligned}
C=\frac{2}{\ln 2}[ & \sum_{i=0}^{N_{r}-1} \frac{(-1)^{i-1} e^{\frac{1}{\gamma}} \mathrm{E}_{i}\left(-\frac{1}{\bar{\gamma}}\right)}{i ! \bar{\gamma}^{i}} \\
& \left.+\sum_{i=0}^{N_{r}-1} \sum_{k=1}^{i} \frac{(k-1) !(-1)^{i-k} \bar{\gamma}^{k}}{i ! \bar{\gamma}^{i}}\right],
\end{aligned}
$$

trong đó $\mathrm{E}_{i}$ là hàm tích phân mũ bậc $i$ được định nghĩa trong [27].

Chứng minh: Để đơn giản, ta giả sử mô hình xem xét là mô hình đối xứng (symmetric), tức là hai thiết bị đầu cuối $\mathrm{A}$ và $\mathrm{B}$ có các tham số giống hệt nhau với $\bar{\gamma}_{\mathrm{A}}=\bar{\gamma}_{\mathrm{B}}=\bar{\gamma}$, $F_{\mathrm{A}}(x)=F_{\mathrm{B}}(x), N_{r}^{\mathrm{A}}=N_{r}^{\mathrm{B}}=N_{r}$. Như vậy (19) được biểu diễn lại thành

$$
C=\frac{2}{\ln 2} \int_{0}^{\infty} \frac{1-F_{\mathrm{A}}(x)}{1+x} d x .
$$

Thay $F_{\mathrm{A}}(x)$ trong (21) bởi $\mathcal{P}_{\mathrm{out}}^{\mathrm{A}}$ trong (7) và áp dụng công thức (3.353.5) trong [27] ta thu được kết quả ở biểu thức (20). Trường hợp mô hình bất đối xứng, biểu thức dung lượng có thể dễ dàng xác định được bằng phương pháp tương tự.

\section{KẾT QUẢ MÔ PHỎNG VÀ THẢO LUẬN}

Trên cơ sở kết quả phân tích trong Mục III, trong phần này phẩm chất hệ thống SM-FD sẽ được biểu diễn thông qua các tham số đánh giá.

Ở đây, chúng tôi xem xét công suất phát, phương sai tạp âm và nhiễu dư tại hai thiết bị đầu cuối là như nhau. Do vậy SNR là tỉ số giữa công suất phát tại một đầu cuối trên phương sai tạp âm, tức là $\mathrm{SNR}=\frac{P_{\mathrm{A}}}{\sigma^{2}}=\frac{P_{\mathrm{B}}}{\sigma^{2}}=\frac{P}{\sigma^{2}}$. Đồng thời, chúng tôi sử dụng mô phỏng Monte Carlo để kiểm chứng kết quả tính toán. Các tham số cho đánh giá, phân tích như sau: độ lợi trung bình của kênh truyền là 1 ; phương sai tạp âm AWGN: $\sigma^{2}=1$, số ăng-ten phát và thu $N_{t}^{\mathrm{A}}=N_{t}^{\mathrm{B}}=N_{t}, N_{r}^{\mathrm{A}}=N_{r}^{\mathrm{B}}=N_{r}$. Nhiễu dư RSI được thay đổi để khảo sát ảnh hưởng của nó đến phẩm chất hệ thống.

Hình 2 cho thấy ảnh hưởng của số lượng ăng-ten thu phát đến xác suất dừng của hệ thống SM-FD trong trường hợp $\mathcal{R}=3 \mathrm{bit} / \mathrm{s} / \mathrm{Hz} ; \tilde{\Omega}=-10 \mathrm{~dB}$, trong đó đường biểu diễn kết quả phân tích lý thuyết sử dụng công thức (8) trong định lý 1. Các ký hiệu đánh dấu (marker symbol) thể hiện kết quả mô phỏng Monte-Carlo. Chú ý rằng, đường lý thuyết cho hệ thống SM-FD sử dụng công thức (8) với $\bar{\gamma}_{\mathrm{A}}=\bar{\gamma}_{\mathrm{B}}=\bar{\gamma}=\frac{P}{\sigma^{2}+\sigma_{\mathrm{RSI}}^{2}}$, trong khi đường lý thuyết cho hệ

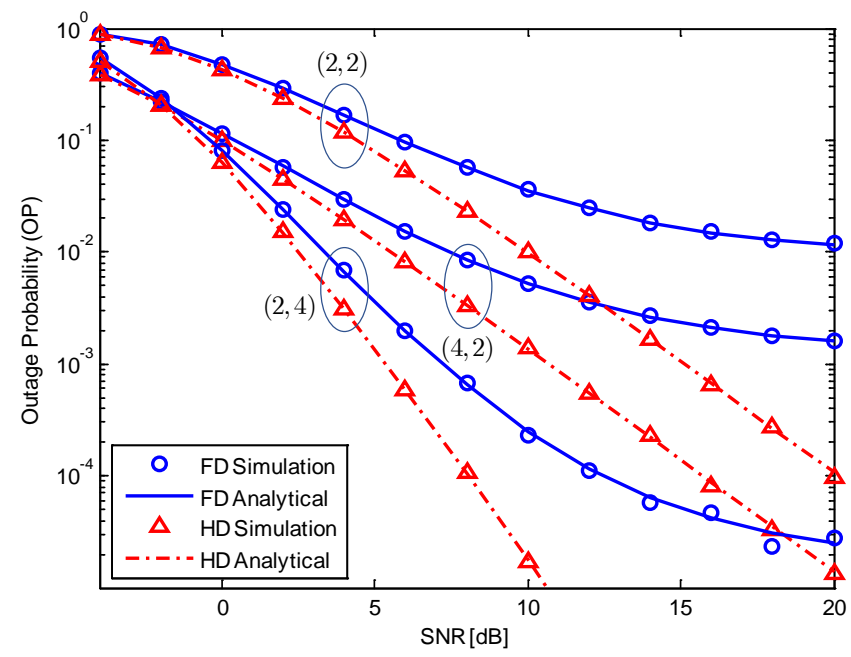

Hình 2. Xác suất dừng hệ thống theo SNR trung bình với số lượng ăng-ten thu phát $\left(N_{t}, N_{r}\right)$ khác nhau; $\mathcal{R}=3 \mathrm{bit} / \mathrm{s} / \mathrm{Hz}$; $\tilde{\Omega}=-10 \mathrm{~dB}$.

thống SM-HD cũng áp dụng công thức này với $\mathrm{RSI}=0$, tức là $\bar{\gamma}=\frac{P}{\sigma^{2}}$.

Sự phù hợp của kết quả phân tích với kết quả mô phỏng cho thây sự đúng đắn của định lý 1 . Dễ dàng nhận thấy rằng, phẩm chất hệ thống SM-FD bị ảnh hưởng mạnh bởi RSI, nhất là vùng SNR cao. Xác suất dừng tiến đến vùng bão hòa do công suất phát càng cao thì RSI càng lớn (vì RSI được xác định bởi $\sigma_{\mathrm{RSI}}^{2}=\tilde{\Omega} P$ ). Mặt khác, khi tăng số ăng-ten phát, phẩm chất xác suất dừng của hệ thống sẽ được cải thiện do số bit truyền đi trên miền ăng-ten tăng lên trong khi số bit truyền qua symbol điều chế giảm xuống. Ví dụ, tại SNR $=14 \mathrm{~dB}$, hệ thống SM-FD cấu hình $(2,2)$ đạt $\mathrm{OP}=10^{-1}$ trong khi cấu hình $(4,2)$ đạt $\mathrm{OP}=3 \times 10^{-2}$, tức là độ lợi mà $\mathrm{SM}$ đem lại khi tăng số ăng-ten phát lên trong trường hợp này là hơn 3 lần. Khi tổng sô ăngten thu và ăng-ten phát không đổi, ví dụ $N_{t}+N_{r}=6$, cấu hình $(2,4)$ cho phẩm chất tốt hơn nhiều so với cấu hình $(4,2)$ do hệ thống SM không có phân tập phát, chỉ có phân tập thu. Ví dụ, hệ thống SM-FD cấu hình $(4,2)$ đạt $\mathrm{OP}=10^{-2}$ tại $\mathrm{SNR}=10 \mathrm{~dB}$ trong khi cấu hình $(2,4)$ không thể đạt đến $\mathrm{OP}=10^{-2}$ do hiện tượng bão hòa.

Hình 3 khảo sát ảnh hưởng của RSI đến xác suất lỗi hệ thống SM-FD tại đầu cuối $\mathrm{B}$, trong đó, đường biểu diễn kết quả phân tích sử dụng công thức (13) ở Định lý 2 với điều chế BPSK. Ở đây số ăng-ten thu phát là $N_{t}=2, N_{r}=4$. Hình 3 cho thây rằng, khi RSI nhỏ $(\tilde{\Omega}=-30 \mathrm{~dB})$, xác suất lỗi của hệ thống SM-FD và SM-HD là như nhau. Tức là trong trường hợp này, có thể nói quá trình SIC của thiết bị FD đã thực hiện lý tưởng. Tuy nhiên, khi nhiễu dư tăng lên, chẳng hạn trường hợp $\tilde{\Omega}=-10 \mathrm{~dB}$ thì phẩm chất hệ thống SM-FD bắt đầu giảm so với hệ thống SM-HD. Khi $\mathrm{SEP}=10^{-4}$ thì độ lợi của hệ thống SM-HD so với SM- 


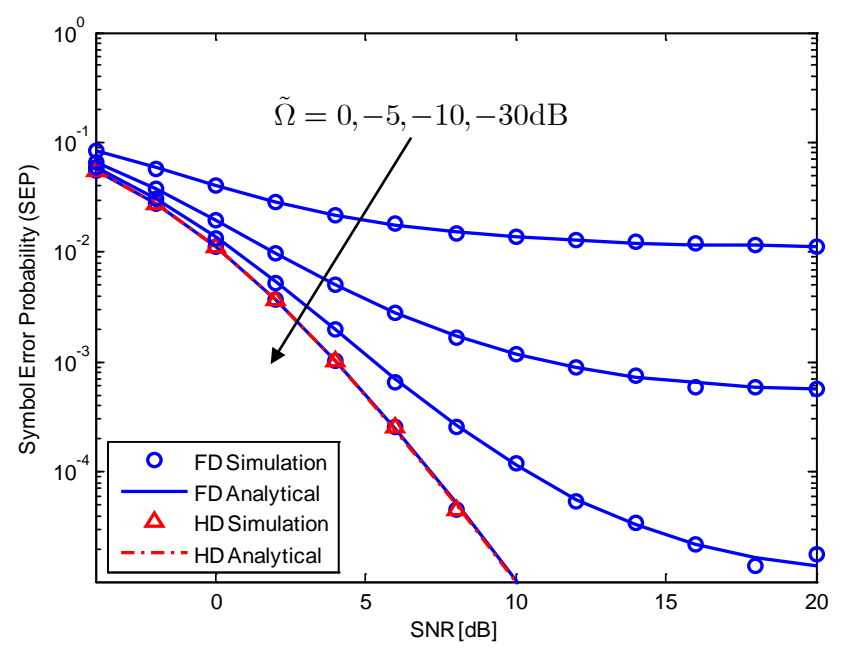

Hình 3. Ảnh hưởng của RSI đến xác suất lỗi hệ thống SM-FD, $N_{t}=2, N_{r}=4$.

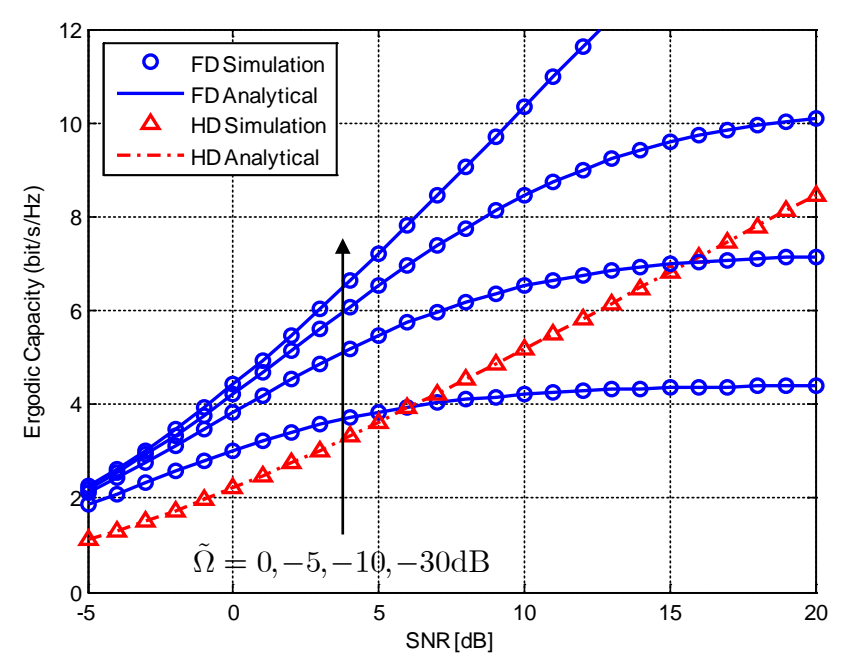

Hình 4. Dung lượng ergodic của hệ thống SM-FD khi so sánh với hệ thống SM-HD với các giá trị RSI khác nhau, $N_{t}=2, N_{r}=4$.

$\mathrm{FD}(\tilde{\Omega}=-10 \mathrm{~dB})$ là khoảng $3 \mathrm{~dB}$. Mặt khác với giá trị RSI này, hệ thống SM-FD không thể đạt $\mathrm{SEP}=10^{-5}$ cho dù công suất phát tiếp tục tăng lên. Khi RSI tăng lên hơn nữa $(\tilde{\Omega}=-5,0 \mathrm{~dB})$ thì phẩm chất hệ thống khảo sát đạt bão hòa tại $\mathrm{SEP}=10^{-3}$ khi $\tilde{\Omega}=-5 \mathrm{~dB}$ và $\mathrm{SEP}=10^{-2}$ khi $\tilde{\Omega}=0 \mathrm{~dB}$.

Hình 4 minh họa dung lượng hệ thống SM-FD khi so sánh với hệ thống SM-HD với các giá trị RSI khác nhau. Với những tham số đã chọn, khi nhiễu dư nhỏ ( $\tilde{\Omega}=-30,-10 \mathrm{~dB})$ dung lượng hệ thống SM-FD luôn tốt hơn so với hệ thống SM-HD. Tuy nhiên, khi nhiễu dư tăng lên thì tùy thuộc vào giá trị SNR để xem xét hệ thống nào có dung lượng cao hơn. Chẳng hạn trong trường hợp $\tilde{\Omega}=-5 \mathrm{~dB}$, khi SNR $<15 \mathrm{~dB}$ thì hệ thống SM-FD cho dung lượng cao hơn hệ thống SM-HD. Nhưng khi tiếp tục tăng SNR, tức là $\mathrm{SNR}>15 \mathrm{~dB}$ thì hệ thống SM-HD lại cho dung lượng cao hơn hệ thống khảo sát. Nguyên nhân là do hệ thống khảo sát đã đạt đến dung lượng bão hòa, đây là kết quả của việc triệt nhiễu không hoàn hảo. Kết hợp các hình 3 và 4 ta thấy rằng, trong trường hợp triệt nhiễu gần như hoàn hảo $(\tilde{\Omega}=-30 \mathrm{~dB})$ thì phẩm chất hệ thống $\mathrm{SM}$ FD và $S M-H D$ là như nhau, trong khi dung lượng hệ thống SM-FD đạt gần gấp đôi dung lượng hệ thống SM-HD. Ví du xét tại $\mathrm{SNR}=10 \mathrm{~dB}$, thì $C_{\mathrm{SM}-\mathrm{FD}}=10,33 \mathrm{bit} / \mathrm{s} / \mathrm{Hs}$ còn $C_{\mathrm{SM}-\mathrm{HD}}=5,18 \mathrm{bit} / \mathrm{s} / \mathrm{Hs}$. Khi nhiễu dư lớn hơn, ví dụ trong trường hợp $\tilde{\Omega}=-10 \mathrm{~dB}$ thì tùy thuộc vào yêu cầu hệ thống để xem xét nên sử dụng chế độ FD hay HD. Chẳng hạn hệ thống cần dung lượng cao và phẩm chất vừa phải thì ta chọn chế độ FD. Ngược lại, hệ thống cần phẩm chất tốt và dung lượng vừa phải thì ta chọn chế độ $\mathrm{HD}$.

\section{KẾT LUẬN}

SM-FD là giải pháp truyền dẫn hứa hẹn cho các hệ thống truyền thông vô tuyến MIMO, đặc biệt là trong kỷ nguyên khan hiếm phổ tần hiện nay. Tuy nhiên, nhiễu SI là yếu tố có ảnh hưởng mạnh đến phẩm chất hệ thống. Bằng phương pháp giải tích, chúng tôi đã đánh giá phẩm chất hệ thống khi có tác động của nhiễu dư do triệt nhiễu không hoàn hảo dưới dạng xác suất dừng, xác suất lỗi ký hiệu và dung lượng hệ thống khi so sánh với hệ thống SM-HD truyền thống. Kết quả phân tích đã chỉ rõ khi mức nhiễu dư nhỏ, dung lượng hệ thống SM-FD đạt gần gấp đôi so với hệ thống SM-HD truyền thống trong khi chất lượng gần như không đổi. Khi mức nhiễu dư lớn hơn, phẩm chất hệ thống SM-FD giảm so với hệ thống SM-HD trong khi dung lượng vẫn cao hơn tùy thuộc vào giá trị cụ thể của RSI và tỉ số tín hiệu trên tạp âm. Đây là nội dung tham khảo quan trọng trong việc lựa chọn chế độ FD hay HD cho hệ thống SM tùy thuộc vào từng điều kiện cụ thể. Tuy nhiên, để tập trung đánh giá mức độ ảnh hưởng của nhiễu SI đến hiệu năng hệ thống, bài báo đã giả thiết máy thu tách chính xác chỉ số ăng-ten phát. Trong thực tế lỗi tách chỉ số ăng-ten phát sẽ gây ảnh hưởng đến hiệu năng chung của hệ thống, vì vậy cần phải được phân tích chi tiết hơn. Kết quả này sẽ được chúng tôi tiến hành trong một nghiên cứu tiếp theo.

\section{TÀI LIỆU THAM KHẢO}

[1] M.-T. Le, V.-D. Ngo, H.-A. Mai, X. N. Tran, and M. Di Renzo, "Spatially modulated orthogonal space-time block codes with non-vanishing determinants," IEEE Transactions on Communications, vol. 62, no. 1, pp. 85-99, 2013.

[2] C. R. Kumar and R. K. Jeyachitra, "Low complexity led grouping based precoding-aided spatial modulation for highly correlated large-scale mimo-vlc channels," IET Communications, vol. 13, no. 3, pp. 312-321, 2018.

[3] R. Y. Mesleh, H. Haas, S. Sinanovic, C. W. Ahn, and S. Yun, "Spatial modulation," IEEE Transactions on vehicular technology, vol. 57, no. 4, pp. 2228-2241, 2008. 
[4] L. Wu, J. Cheng, Z. Zhang, J. Dang, and H. Liu, "Lowcomplexity spatial modulation for im/dd optical wireless communications," IEEE Photonics Technology Letters, vol. 31, no. 6, pp. 475-478, 2019.

[5] D.-T. Phan-Huy, Y. Kokar, K. Rachedi, P. Pajusco, A. Mokh, T. Magounaki, R. Masood, C. Buey, P. Ratajczak, N. Malhouroux-Gaffet et al., "Single-carrier spatial modulation for the internet of things: Design and performance evaluation by using real compact and reconfigurable antennas," IEEE access, vol. 7, pp. 18 978-18993, 2019.

[6] M. Maleki, K. Mohamed-Pour, and M. Soltanalian, "Receive spatial modulation in correlated massive mimo with partial csi," IEEE Transactions on Signal Processing, vol. 67, no. 5, pp. 1237-1250, 2018.

[7] H. S. Hussein, M. Elsayed, U. S. Mohamed, H. Esmaiel, and E. M. Mohamed, "Spectral efficient spatial modulation techniques," IEEE Access, vol. 7, pp. 1454-1469, 2018.

[8] C. Li, Z. Chen, Y. Wang, Y. Yao, and B. Xia, "Outage analysis of the full-duplex decode-and-forward two-way relay system," IEEE Trans. Veh. Technol., vol. 66, no. 5, pp. 4073-4086, May 2017.

[9] A. Almradi and K. A. Hamdi, "Mimo full-duplex relaying in the presence of co-channel interference," IEEE Transactions on Vehicular Technology, vol. 66, no. 6, pp. 4874-4885, 2016.

[10] B. C. Nguyen, X. N. Tran, and D. T. Tran, "Performance analysis of in-band full-duplex amplify-and-forward relay system with direct link," in 2018 2nd International Conference on Recent Advances in Signal Processing, Telecommunications \& Computing (SigTelCom). IEEE, 2018, pp. 192-197.

[11] B. C. Nguyen and X. N. Tran, "Performance analysis of full-duplex amplify-and-forward relay system with hardware impairments and imperfect self-interference cancellation," Wireless Communications and Mobile Computing, vol. 2019, 2019.

[12] X. N. Tran, B. C. Nguyen, and D. T. Tran, "Outage probability of two-way full-duplex relay system with hardware impairments," in 2019 3rd International Conference on Recent Advances in Signal Processing, Telecommunications \& Computing (SigTelCom). IEEE, 2019, pp. 135-139.

[13] B. C. Nguyen, X. N. Tran, T. M. Hoang et al., "Performance analysis of full-duplex vehicle-to-vehicle relay system over double-rayleigh fading channels," Mobile Networks and Applications, pp. 1-10, 2019.

[14] B. Jiao, M. Wen, M. Ma, and H. V. Poor, "Spatial modulated full duplex," IEEE Wireless Communications Letters, vol. 3, no. 6, pp. 641-644, 2014

[15] A. Koc, I. Altunbas, and E. Basar, "Two-way full-duplex spatial modulation systems with wireless powered af relaying," IEEE Wireless Communications Letters, vol. 7, no. 3, pp. 444-447, 2017.

[16] S. Narayanan, H. Ahmadi, and M. F. Flanagan, "On the performance of spatial modulation mimo for full-duplex relay networks," IEEE Transactions on Wireless Communications, vol. 16, no. 6, pp. 3727-3746, 2017.

[17] P. Raviteja, Y. Hong, and E. Viterbo, "Spatial modulation in full-duplex relaying," IEEE Communications Letters, vol. 20, no. 10, pp. 2111-2114, 2016.

[18] M. Duarte, C. Dick, and A. Sabharwal, "Experimentdriven characterization of full-duplex wireless systems," IEEE Transactions on Wireless Communications, vol. 11, no. 12 , pp. 4296-4307, 2012.

[19] E. Everett, A. Sahai, and A. Sabharwal, "Passive self-interference suppression for full-duplex infrastructure nodes," IEEE Transactions on Wireless Communications, vol. 13, no. 2, pp. 680-694, 2014.
[20] D. Bharadia, E. McMilin, and S. Katti, "Full duplex radios," in Proceedings of the ACM SIGCOMM 2013 Conference, ser. SIGCOMM '13. New York, NY, USA: ACM, 2013, pp. 375-386.

[21] T. Riihonen, S. Werner, and R. Wichman, "Hybrid fullduplex/half-duplex relaying with transmit power adaptation," IEEE Trans. Wireless Commun., vol. 10, no. 9, pp. 30743085, Sept. 2011.

[22] E. Ahmed and A. M. Eltawil, "All-digital self-interference cancellation technique for full-duplex systems," IEEE Transactions on Wireless Communications, vol. 14, no. 7, pp. 3519-3532, 2015.

[23] A. Goldsmith, Wireless communications. Cambridge university press, 2005.

[24] R. Rajashekar, K. Hari, and L. Hanzo, "Antenna selection in spatial modulation systems," IEEE Communications Letters, vol. 17, no. 3, pp. 521-524, 2013.

[25] F. Yarkin and I. Altunbas, "Outage performance of spatial modulation with transmit antenna selection over nakagami-m fading channels with arbitrary m," in 2016 8th International Congress on Ultra Modern Telecommunications and Control Systems and Workshops (ICUMT). IEEE, 2016, pp. 438442.

[26] A. Bhowal and R. S. Kshetrimayum, "Outage probability bound of decode and forward two-way full-duplex relay employing spatial modulation over cascaded $\alpha$ - $\mu$ channels," International Journal of Communication Systems, vol. 32, no. 3, p. e3876, 2019.

[27] A. Jeffrey and D. Zwillinger, Table of integrals, series, and products. Academic press, 2007.

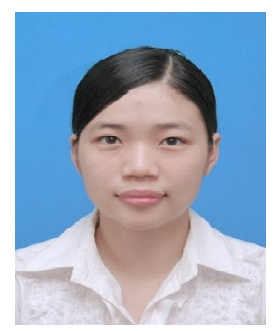

Nguyễn Lê Vân Nhận bằng Kỹ sư và Thạc sỹ tại Học viện Kỹ thuật Quân sự năm 2008 và 2010 . Hiện đang là nghiên cứu sinh tại Khoa Vô tuyến Điện tử, Học viện Kỹ thuật Quân sự. Lĩnh vực nghiên cứu bao gồm MIMO, điều chế không gian, truyền thông song công.

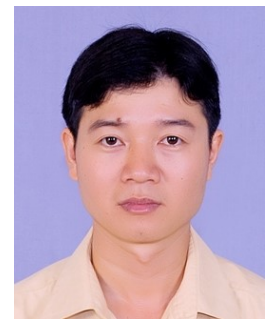

Nguyễn Bá Cao Tốt nghiệp trường Sĩ quan Thông tin năm 2006, nhận bằng Thạc sĩ tại Học viện Công nghệ Bưu chính Viễn thông năm 2011. Hiện tại là nghiên cứu sinh tại Khoa Vô tuyến điện tử, Học viện Kỹ thuật Quân sự. Lĩnh vực nghiên cứu bao gồm truyền thông song công, truyền thông hợp tác. 


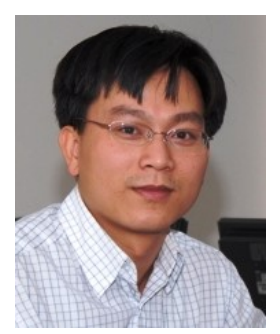

Trân Xuân Nam Nhận bằng Thạc sỹ năm 1998 tại Trương Đại học Công nghệ, Sydney, Úc. Nhận bằng Tiến sỹ năm 2003 tại Trường Đại học Điện tử - Truyền thông, Tokyo, Nhật Bản. Tác giả đang là phó giáo sư chủ trì Nhóm nghiên cứu mạnh về Thông tin vô tuyến tiên tiến (awc.lqdtu.edu.vn) của Học viện Kỹ thuật Quân sự. Tác giả đã được tặng Giải thưởng nhà nghiên cứu trẻ IEEE AP-S tại Nhật Bản năm 2003, Giải thưởng Bài báo xuất sắc nhất tại Hội nghị quốc tế năm 2012 về Các công nghệ tiên tiến trong truyền thông (ATC 2012), Giải thưởng bài báo xuất sắc tại Hội nghị Quốc gia năm 2014 về Điện tử, Truyền thông và Công nghệ thông tin (ECIT 2014). Tác giả là thành viên của IEEE, IEICE và Hội Vô tuyến điện tử Việt Nam. Lĩnh vực nghiên cứu bao gồm ăng-ten thích ứng, xử lý tín hiệu, mã hóa và hệ thống MIMO. 